\title{
Front Matter: Volume 6999
}

, "Front Matter: Volume 6999," Proc. SPIE 6999, Organic Optoelectronics and Photonics III, 699901 (20 May 2008); doi: 10.1117/12.802145

SPIE. Event: SPIE Photonics Europe, 2008, Strasbourg, France 


\title{
PROCEEDINGS OF SPIE
}

\section{Organic Optoelectronics and Photonics III}

\author{
Paul L. Heremans \\ Michele Muccini \\ Eric A. Meulenkamp \\ Editors
}

\section{7-10 April 2008 \\ Strasbourg, France}

Sponsored by

SPIE Europe

Cosponsored by

Alsace International (France)

Conseil Général du Bas-Rhin (France)

Région Alsace (France)

Communauté Urbaine de Strasbourg (France)

Cooperating Organizations

AFOP_Association Française des Industries de l'Optique et de la Photonique (France)

EOS-European Optical Society (Germany) • EPIC-European Photonics Industry Consortium

(France) • ePIXnet (Belgium) • IOP-Institute of Physics (United Kingdom) • NEMO-Network of

Excellence on Micro-Optics (Belgium) • OLAS (Italy) • The OLLA Project (Germany) • OPERA 2015

(Belgium) • PhOREMOST (Ireland) • Photonics Knowledge Transfer Network (United Kingdom)

Photonics Cluster (United Kingdom) • Photonics4Life (Germany) • Photonics 21 (Germany)

RhenaPhotonics Alsace (France)

Published by

SPIE

Volume 6999 
The papers included in this volume were part of the technical conference cited on the cover and title page. Papers were selected and subject to review by the editors and conference program committee. Some conference presentations may not be available for publication. The papers published in these proceedings reflect the work and thoughts of the authors and are published herein as submitted. The publisher is not responsible for the validity of the information or for any outcomes resulting from reliance thereon.

Please use the following format to cite material from this book:

Author(s), "Title of Paper," in Organic Optoelectronics and Photonics III, edited by Paul L. Heremans, Michele Muccini, Eric A. Meulenkamp, Proceedings of SPIE Vol. 6999 (SPIE, Bellingham, WA, 2008) Article CID Number.

ISSN 0277-786X

ISBN 9780819471970

Published by

SPIE

P.O. Box 10, Bellingham, Washington 98227-0010 USA

Telephone +1 3606763290 (Pacific Time) · Fax +1 3606471445

SPIE.org

Copyright (c) 2008, Society of Photo-Optical Instrumentation Engineers

Copying of material in this book for internal or personal use, or for the internal or personal use of specific clients, beyond the fair use provisions granted by the U.S. Copyright Law is authorized by SPIE subject to payment of copying fees. The Transactional Reporting Service base fee for this volume is $\$ 18.00$ per article (or portion thereof), which should be paid directly to the Copyright Clearance Center (CCC), 222 Rosewood Drive, Danvers, MA 01923. Payment may also be made electronically through CCC Online at copyright.com. Other copying for republication, resale, advertising or promotion, or any form of systematic or multiple reproduction of any material in this book is prohibited except with permission in writing from the publisher. The CCC fee code is 0277-786X/08/\$18.00.

Printed in the United States of America.

Publication of record for individual papers is online in the SPIE Digital Library.

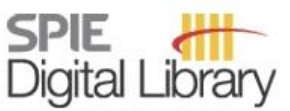

SPIEDigitalLibrary.org

Paper Numbering: Proceedings of SPIE follow an e-First publication model, with papers published first online and then in print and on CD-ROM. Papers are published as they are submitted and meet publication criteria. A unique, consistent, permanent citation identifier (CID) number is assigned to each article at the time of the first publication. Utilization of CIDs allows articles to be fully citable as soon they are published online, and connects the same identifier to all online, print, and electronic versions of the publication. SPIE uses a six-digit CID article numbering system in which:

- The first four digits correspond to the SPIE volume number.

- The last two digits indicate publication order within the volume using a Base 36 numbering system employing both numerals and letters. These two-number sets start with $00,01,02,03,04,05$, 06, 07, 08, 09, OA, OB ... 0Z, followed by 10-1Z, 20-2Z, etc.

The CID number appears on each page of the manuscript. The complete citation is used on the first page, and an abbreviated version on subsequent pages. Numbers in the index correspond to the last two digits of the six-digit CID number. 


\section{Contents}

xi Conference Committee

\section{LASERS I}

699902 Sub-nanojule threshold lasing in $5 \times 5 \mu^{2}$ organic photonic boxes (Invited Paper) [6999-01] M. Langner, M. Sudzius, S. I. Hintschich, H. Fröb, V. G. Lyssenko, K. Leo, Technische Univ. Dresden (Germany)

\section{LASERS II}

699906 Ultra-small footprint photonic crystal lasers with organic gain material (Invited Paper) [6999-05]

K. Baumann, N. Moll, T. Stöferle, IBM Research (Switzerland); T. Wahlbrink, J. Bolten, T. Mollenhaver, C. Moormann, AMO GmbH (Germany); B. Wang, U. Scherf, Bergische Univ. Wuppertal (Germany); R. F. Mahrt, IBM Research (Switzerland)

699907 Blue-light-emitting polymer lasers with non-periodic circular Bragg resonators [6999-06] T. Wellinger, Imperial College London (United Kingdom); C. Pflumm, Merck KGaA (Germany); J. Becker, T. Weimann, Physikalisch-Technische Bundesanstalt (Germany); M. Campoy-Quiles, P. N. Stavrinou, Imperial College London (United Kingdom); U. Scherf, Bergische Univ. Wuppertal (Germany); D. D. C. Bradley, Imperial College London (United Kingdom)

699908 Measurement of triplet exciton diffusion in the context of organic lasers [6999-07] H. Choukri, M. Lebental, S. Forget, S. Chénais, D. Adès, A. Siove, Institut Galilée, Univ. Paris-Nord (France); B. Geffroy, CEA-LITEN (France)

\section{PHOTOPHYSICS II}

6999 OC Theory of directed transportation of electronic excitation between single molecules through photonic coupling [6999-12]

D. L. Andrews, D. S. Bradshaw, Univ. of East Anglia, Norwich (United Kingdom)

\section{NONLINEAR OPTIC MATERIALS}

6999 0l Generation of microstructures in novel supramolecular ionic materials based on azobenzene [6999-18]

O. Kulikovska, Fraunhofer Institute for Applied Polymer Research (Germany); L. Kulikovsky, L. M. Goldenberg, Institute of Thin Film Technology and Microsensorics (Germany); J. Stumpe, Fraunhofer Institute for Applied Polymer Research (Germany) and Institute of Thin Film Technology and Microsensorics (Germany) 
6999 0J Multipolar dyes for NLO: solvation and aggregation effects [6999-19]

F. Terenziani, C. Sissa, A. Painelli, Parma Univ. (Italy)

6999 OK One- and two-photon absorption and emission properties of heteroaromatic bichromophores [6999-20]

A. Abbotto, L. Bellotto, Univ. of Milano-Bicocca (Italy); R. Bozio, C. Ferrante, I. Fortunati,

E. Garbin, Univ. of Padova (Italy); C. Marinzi, Univ. of Milano-Bicocca (Italy); A. Painelli,

C. Sissa, F. Terenziani, Univ. of Parma (Italy)

6999 OM Polyfluorene-PMMA copolymers for plastic optical fibers with gain [6999-22]

J. Morgado, A. L. Mendonça, A. Charas, Instituto Superior Técnico (Portugal); J. Clark,

G. Lanzani, Politecnico di Milano (Italy); L. Bazzana, A. Nocivelli, Luceat S.p.A. (Italy)

\section{ORGANIC PHOTOVOLTAICS III: JOINT SESSION WITH CONFERENCE 7002}

699900 Ruthenium sensitizers based on heteroaromatic conjugated bypiridines for dye-sensitized solar cells [6999-24]

A. Abbotto, Univ. of Milano-Bicocca (Italy); C. Barolo, J.-H. Yum, Swiss Federal Institute of Technology (Switzerland); L. Bellotto, Univ. of Milano-Bicocca (Italy); F. De Angelis, Istituto CNR di Scienze e Tecnologie Molecolari, Univ. of Perugia (Italy); M. Grätzel, Swiss Federal Institute of Technology (Switzerland); C. Marinzi, Univ. of Milano-Bicocca (Italy); M. K. Nazeeruddin, Swiss Federal Institute of Technology (Switzerland)

6999 OS 1,4,5,8-Naphthalenetetracarboxylic dianhydride as transparent electron transport material in organic p-i-n solar cells [6999-28]

C. Falkenberg, C. Uhrich, B. Maennig, M. K. Riede, K. Leo, TU Dresden (Germany)

\section{PHOTODETECTORS}

6999 OW High-speed organic photo-detectors fabricated by vacuum and solution processes and application for optical transmission [6999-32]

Y. Ohmori, T. Hamasaki, Osaka Univ. (Japan); T. Morimune, Takuma National College of Technology (Japan); H. Kajii, Osaka Univ. (Japan)

6999 OX Light-sensing ambipolar organic transistors for optoelectronic applications [6999-33] T. D. Anthopoulos, P. H. Wöbkenberg, D. D. C. Bradley, Imperial College London (United Kingdom)

\section{LIGHT-EMITTING DETECTORS}

$69990 Z$ Fabrication of ambipolar light-emitting transistor using high-photoluminescent organic single crystal [6999-35]

S. Z. Bisri, Tohoku Univ. (Japan); T. Takenobu, Tohoku Univ. (Japan) and CREST, Japan Science and Technology Corp. (Japan); Y. Yomogida, Tohoku Univ. (Japan); T. Yamao, Kyoto Institute of Technology (Japan); M. Yahiro, Kyushu Univ. (Japan); S. Hotta, Kyoto Institute of Technology (Japan); C. Adachi, Kyushu Univ. (Japan); Y. Iwasa, Tohoku Univ. (Japan) and CREST, Japan Science and Technology Corp. (Japan) 
699910 Performance of organic light-emitting diodes with remote metallic contact using high mobility electron-transport layers [6999-36]

S. Schols, IMEC VZW (Belgium) and Katholieke Univ. Leuven (Belgium); C. McClatchey, Queen's Univ. Belfast (United Kingdom); J. Genoe, IMEC Vzw (Belgium); P. Heremans, IMEC Vzw (Belgium) and Katholieke Univ. Leuven (Belgium); A. Facchetti, Northwestern Univ. (USA)

\section{OLED PHYSICS I}

699912 Spin and device engineering for blue organic light emitting devices (Invited Paper) [6999-38]

M. Segal, C. Mulder, K. Celebi, M. Singh, K. Rivoire, S. Difley, T. Van Voorhis, M. A. Baldo, Massachusetts Institute of Technology (USA)

699913 Influence of a magnetic field on the device performance of OLEDs [6999-39]

U. Niedermeier, Siemens AG (Germany) and Technische Univ. of Darmstadt (Germany); W. Sarfert, Siemens AG (Germany); H. von Seggern, Technische Univ. of Darmstadt (Germany)

\section{OLED PHYSICS II}

699917 Efficiency and lifetime enhancement of phosphorescent organic devices [6999-43] R. Meerheim, S. Scholz, G. Schwartz, S. Reineke, S. Olthof, K. Walzer, K. Leo, Technische Univ. Dresden (Germany)

699918 Analysis of charge transport path ways in disordered organic conductors [6999-44] W. Wenzel, Forschungszentrum Karlsruhe, Institut für Nanotechnologie (Germany); J. J. Kwiatkowski, J. Nelson, Imperial College, London (United Kingdom); H. Li, J. L. Bredas, Georgia Institute of Technology (USA); C. Lennartz, BASF AG (Germany)

699919 A theoretical view on self-assembled monolayers in organic electronic devices (Invited Paper) [6999-45]

G. Heimel, Humboldt Univ. zu Berlin (Germany) and Georgia Institute of Technology (USA); L. Romaner, Univ. of Leoben (Austria); E. Zojer, Graz Univ. of Technology (Austria); J.-L. Brédas, Georgia Institute of Technology (USA)

\section{OLED MATERIALS AND TECHNOLOGY I}

6999 1B Chemical degradation mechanisms of organic semiconductor devices [6999-47]

S. Scholz, R. Meerheim, K. Walzer, K. Leo, Technische Univ. Dresden (Germany)

6999 1C High efficient pin orange organic light emitting diode fabrication with novel Al cathode using DC magnetron sputtering [6999-48]

T. H. Gil, S. Franke, C. May, J. Amelung, H. Lakner, K. Leo, Fraunhofer-Institut für Photonische Microsysteme (Germany) 
6999 IE An overview about the use of electrical doping of charge carrier transport layers in OLEDs and further organic electronic applications (Invited Paper) [6999-50]

S. Madasamy, D. Pavicic, C. Rothe, S. Murano, J. Birnstock, J. Blochwitz-Nimoth, Novaled AG (Germany); S. Mladenovski, K. Neyts, Ghent Univ. (Belgium); M. Pfeiffer, Heliatek GmbH (Germany)

699911 Multilayer hybrid LEDs based on colloidal inorganic semiconductors nanocrystal and PIN technology [6999-54]

A. Rizzo, M. Mazzeo, G. Gigli, National Nanotechnology Lab. of CNR-INFM, Univ. del Salento (Italy)

\section{POSTER SESSION}

$69991 \mathrm{~J}$ Enlarged bilayer interfaces from liquid-liquid dewetting for photovoltaic applications [6999-55]

J. Heier, EMPA (Switzerland); J. Groenewold, Univ. of Utrecht (Netherlands); F. A. Castro,

F. Nüesch, R. Hany, EMPA (Switzerland)

$69991 \mathrm{~K} \quad$ Electroabsorption study of the influence of PEDOT:PSS on organic solar cells performances [6999-56]

J. Navarro, H. Kanaan, I. Seguy, P. Jolinat, P. Destruel, G. Ablart, Univ. Paul Sabatier (France); H. Bock, CNRS, Univ. Bordeaux I (France)

6999 1L Using optical thin film model to optimize thermal annealing procedure in P3HT:PCBM blend based solar cells [6999-57]

W. H. Lee, H. L. Chen, S. Y. Chuang, T. H. Chen, W. F. Su, National Taiwan Univ. (Taiwan)

$69991 \mathrm{M}$ Characterisation of different hole transport materials as used in organic $p$-i-n solar cells [6999-58]

S. Pfuetzner, A. Petrich, Technische Univ. Dresden (Germany); C. Malbrich, Leibniz-Institut für Festkörper- und Werkstoffforschung Dresden (Germany); J. Meiss, M. Koch, M. K. Riede, Technische Univ. Dresden (Germany); M. Pfeiffer, Heliatek GmbH (Germany); K. Leo, Technische Univ. Dresden (Germany)

6999 iN P3HT/PCBM/SWNTs photovoltaic devices [6999-59]

E. Kymakis, E. Koudoumas, Technological Education Institute of Crete (Greece)

$69991 \mathrm{~V}$ Investigation of the optical waveguide realisation based on polymers with large third order non-linear susceptibility [6999-67]

K. Messaad, D. Bosc, M. Thual, S. Haesaert, F. Henrio, CCLO, CNRS (France)

6999 IW Organic materials for molecular switching [6999-68]

I. Asselberghs, Katholieke Univ. Leuven (Belgium); G. Hennrich, Univ. Autónoma de Madrid (Spain); J. McCleverty, Univ. of Bristol (United Kingdom); L. Boubekeur-Lecaque, B. J. Coe, Univ. of Manchester (United Kingdom); K. Clays, Katholieke Univ. Leuven (Belgium)

6999 1X Analysis of diffraction characteristics of photopolymers by using the FDTD method [6999-69] K. Shimada, S. Yoshida, N. Yoshida, M. Yamamoto, Tokyo Univ. of Science (Japan) 
699912 Research on the growth of dye film in vacuum in situ [6999-71]

K. Gryłsenko, T. Doroshenko, Yu. Kolomzarov, V. Prokopets, O. Fedoriak, R. Zelinski,

O. Lytvyn, Institute of Semiconductor Physics (Ukraine); D. Prescher, B. Grimm, V. Ksianzou,

S. Schrader, Univ. of Applied Sciences (Germany); O. Tolmachev, Yu. Slominskii,

V. Kurdiukov, G. Smirnova, Institute of Organic Chemistry (Ukraine)

699920 Enigma of the second harmonic generation in oriented film of symmetric squaraine [6999-72]

K. Grytsenko, Institute of Semiconductor Physics (Ukraine); Y. Slominski, O. Tolmachev, Institute of Organic Chemistry (Ukraine); R. Resel, Graz Univ. of Technology (Austria);

S. Ksensov, S. Schrader, Univ. of Applied Sciences, Wildau (Germany)

699921 Stable and efficient organo-inorganic emitting materials: a new rare earth-MOF family [6999-73]

F. Gándara, N. Snejko, R. Martínez-Morillas, A. de Andrés, Consejo Superior de Investigaciones Científicas (Spain); C. Coya, Univ. Rey Juan Carlos (Spain);

E. Gutiérrez-Puebla, M. A. Monge, Consejo Superior de Investigaciones Científicas (Spain)

699922 Sum-rules and quantum limits: nonlinear optics from first principles [6999-74]

J. Pérez-Moreno, Univ. of Leuven (Belgium); K. Clays, Univ. of Leuven (Belgium) and Washington State Univ. (USA); M. G. Kuzyk, Washington State Univ. (USA)

699923 Benzathiazoliums and pyridiniums for second-order nonlinear optics [6999-75]

E. Franz, Katholieke Univ. Leuven (Belgium); E. C. Harper, B. J. Coe, Univ. of Manchester (United Kingdom); P. Zahradnik, Comenius Univ. Bratislava (Slovak Republic); K. Clays, I. Asselberghs, Katholieke Univ. Leuven (Belgium)

699924 Influence of corona poling procedures on linear and non-linear optical properties of polymer materials containing indandione derivatives as a cromophores [6999-76] A. Vembris, M. Rutkis, E. Laizane, Univ. of Latvia (Latvia)

699925 Study of the luminescence properties of $\mathrm{Nd}(\mathrm{TTA})_{3}$ phen-doped 6-FDA/epoxy waveguides [6999-77]

J. Yang, M. B. J. Diemeer, L. T. H. Hilderink, M. Pollnau, A. Driessen, Univ. of Twente (Netherlands)

699928 Electro-optical properties of volume holograms in organic conductive materials [6999-80] M. P. Hernández-Garay, A. Olivares-Pérez, R. C. Fontanilla-Urdaneta, I. Fuentes-Tapia, Instituto Nacional de Astrofísica Óptica y Electrónica (Mexico)

6999 2C Electroabsorption spectra of multipolar chromophores [6999-84]

C. Sissa, F. Terenziani, A. Painelli, Univ. degli Studi di Parma (Italy)

6999 2D Emission properties of artificial opals infiltrated with a heteroaromatic quadrupolar dye [6999-108]

M. Cucini, D. Comoretto, Univ. di Genova (Italy); M. Galli, F. Marabelli, Univ. di Pavia (Italy);

A. Abbotto, L. Bellotto, C. Marinzi, Univ. di Milano-Bicocca (Italy)

6999 2G Integration of top-emitting organic light emitting diodes on CMOS substrates [6999-111] M. Toerker, Ch. Grillberger, D. Kreye, U. Vogel, J. Amelung, Fraunhofer-Institute for Photonic Microsystems (Germany) 
$69992 \mathrm{H} \quad$ A fluorescent stilbenoid dendrimer for solution-processed blue light emitting diodes [6999-112]

C. Coya, A. L. Álvarez, M. Ramos, Univ. Rey Juan Carlos (Spain); A. de Andrés, C. Zaldo, Instituto de Ciencia de Materiales de Madrid (Spain); R. Gómez, J. L. Segura, C. Seoane, Univ. Complutense de Madrid (Spain)

699921 Development of printed ITO coatings on PET and PEN foil for flexible organic photodiodes [6999-1 13]

S. Heusing, P. W. Oliveira, INM, Leibniz-Institut für Neue Materialien GmbH (Germany);

E. Kraker, A. Haase, C. Palfinger, JOANNEUM Research Forschungsgesellschaft mbH

(Austria); M. Veith, INM, Leibniz-Institut für Neve Materialien GmbH (Germany)

$69992 \mathrm{~L} \quad$ Unexpected large spectral shift from blue to green region in a light-emitting electrochemical cell [6999-116]

H. J. Bolink, E. Coronado, R. D. Costa, N. Lardiés, Univ. de València (Spain);

Md. K. Nazeeruddin, Ecole Polytechnique Fédérale de Lausanne (Switzerland); E. Ortí, Univ. de València (Spain)

6999 2N Photoluminescence degradation of blue OLED emitters [6999-118]

S. Winter, S. Reineke, K. Walzer, K. Leo, Technische Univ. Dresden (Germany)

$69992 \mathrm{R} \quad$ An effective intermediate Al/Au electrode for stacked color-tunable organic light emitting devices [6999-87]

T. Zheng, W. C. H. Choy, The Univ. of Hong Kong (Hong Kong China)

$699925 \quad H i g h-e f f i c i e n c y$ fluorescent white organic light-emitting diodes using double holetransporting-layers [6999-88]

J.-H. Jou, S.-M. Shen, C.-C. Chen, Y.-C. Chung, C.-J. Wang, M.-F. Hsu, W. Wang, M.-H. Wu, C.-J. Yang, C.-P. Liu, National Tsing Hua Univ. (Taiwan)

6999 2T Light extraction from OLEDS with (high) index matched glass substrates [6999-89]

G. Gaertner, H. Greiner, Philips Research Europe (Germany)

$69992 \mathrm{U}$ Cathode effect on current-voltage characteristics of blue light-emitting diodes based on a polyspirofluorene [6999-90]

G. Garcia-Belmonte, E. M. Barea, Y. Ayyad-Limonge, J. M. Montero, Univ. Jaume I (Spain);

H. J. Bolink, Univ. de Valencia (Spain); J. Bisquert, Univ. Jaume I (Spain)

$69992 \mathrm{~V} \quad$ Light extraction and optical loss mechanisms in organic light-emitting diodes [6999-91] S. Nowy, N. A. Reinke, J. Frischeisen, W. Brütting, Univ. of Augsburg (Germany)

$69992 \mathrm{X}$ Inverted solution processable OLEDs using a metal oxide as electron injection contact [6999-93]

H. J. Bolink, E. Coronado, D. Repetto, M. Sessolo, Univ. de Valencia (Spain)

$69992 Y \quad$ Emission enhancement of microlens on OLED with different layer structures [6999-94]

Y.-H. Ho, National Taiwan Univ. (Taiwan); J.-H. Fang, National Dong Hwa Univ. (Taiwan);

J.-H. Lee, National Taiwan Univ. (Taiwan); M.-K. Wei, H.-Y. Lin, National Dong Hwa Univ.

(Taiwan) 
699930 Lower limit of the lasing threshold in an organic microcavity [6999-96]

B. Schütte, H. Gothe, M. Sudzius, V. G. Lyssenko, S. I. Hintschich, H. Fröb, K. Leo, Technische Univ. Dresden (Germany)

699934 A photophysical study of substituted arylethynylenes [6999-102]

A. Beeby, Durham Univ. (United Kingdom); I. Clark, Rutherford Appleton Lab.

(United Kingdom); K. S. Findlay, Sheffield Univ. (United Kingdom); P. Matousek, Rutherford Appleton Lab. (United Kingdom); L. Porrès, Durham Univ. (United Kingdom); A. W. Parker, Rutherford Appleton Lab. (United Kingdom); S. Rutter, Durham Univ. (United Kingdom);

M. Towrie, Rutherford Appleton Lab. (United Kingdom)

699935 Exciton size and mobility in (6,5) single-walled carbon nanotubes [6999-103]

L. LÜer, S. Hoseinkhani, National Lab. for Ultrafast and Ultraintense Optical Science, INFMCNR, Politecnico di Milano (Italy); D. Polli, CNISM, Politecnico di Milano (Italy); J. Crochet, T. Hertel, Vanderbilt Univ. (USA); G. Lanzani, CNISM, Politecnico di Milano (Italy)

Author Index 
Downloaded From: https://www.spiedigitallibrary.org/conference-proceedings-of-spie on 25 Apr 2023

Terms of Use: https://www.spiedigitallibrary.org/terms-of-use 


\title{
Conference Committee
}

\author{
Symposium Chairs \\ Hugo Thienpont, Vrije Universiteit Brussel (Belgium) \\ Patrick P. Meyrueis, Université Louis Pasteur (France) \\ Giancarlo C. Righini, Istituto di Fisica Applicata Nello Carrara, CNR \\ (Italy) \\ Conference Chairs
}

Paul L. Heremans, IMEC (Belgium)

Michele Muccini, Istituto per lo Studio dei Materiali Nanostrutturati, CNR (Italy)

Eric A. Meulenkamp, Philips Research Laboratories (Netherlands)

Program Committee

Chihaya Adachi, Kyushu University (Japan)

Heinrich Becker, Merck OLED Materials GmbH (Germany)

David Beljonne, Université de Mons-Hainaut (Belgium)

Paul W. M. Blom, Rijksuniv. Groningen (Netherlands)

Herbert F. Boerner, Philips Research Laboratories (Germany)

Donal D. C. Bradley, Imperial College London (United Kingdom)

Franco Cacialli, University College London (United Kingdom)

Gunther Haas, CEA-LETI (France)

Richard H. Friend, University of Cambridge (United Kingdom)

René A. J. Janssen, Technische Universiteit Eindhoven (Netherlands)

Junji Kido, Yamagata University (Japan)

Guglielmo Lanzani, Politecnico di Milano (Italy)

Karl Leo, Technische Universität Dresden (Germany)

Rainer F. Mahrt, IBM Zürich Research Laboratory (Switzerland)

Niyazi S. Sariciftci, Johannes Kepler University Linz (Austria)

Paul van der Schaaf, Ciba Specialty Chemicals (Switzerland)

Uli Lemmer, Universität Karlsruhe (Germany)

\section{Session Chairs}

1 Lasers I

Rainer F. Mahrt, IBM Zürich Research Laboratory (Switzerland)

2 Lasers II

Michele Muccini, Istituto per lo Studio dei Materiali Nanostrutturati,

CNR (Italy) 
3 Photophysics I

Michele Muccini, Istituto per lo Studio dei Materiali Nanostrutturati, CNR (Italy)

4 Photophysics II

Luisa De Cola, Westfälische Wilhelms-Universität Münster (Germany)

5 Sensors and Switches

Eric A. Meulenkamp, Philips Research Laboratories (Netherlands)

$6 \quad$ Nonlinear Optic Materials

Michele Muccini, Istituto per lo Studio dei Materiali Nanostrutturati, CNR (Italy)

7 Organic Photovoltaics III: Joint Session with Conference 7002

Paul L. Heremans, IMEC (Belgium)

8 Photodetectors

Christoph J. Brabec, Konarka Austria (Austria)

$9 \quad$ Light-Emitting Detectors

Reinder Coehoorn, Philips Research Laboratories (Netherlands)

10 OLED Physics I

Joseph Shinar, lowa State University (USA)

11 OLED Physics II

Michael Segal, Massachusetts Institute of Technology (USA)

12 OLED Materials and Technology I

Jan Blochwitz-Nimoth, Novaled GmbH (Germany)

13 OLED Materials and Technology II

Ton Van Mol, TNO (Netherlands) 